\title{
Injection locking of the gyrotropic vortex motion in a nanopillar
}

R. Lehndorff, D. E. Bürgler, C. M. Schneider, and Z. Celinski

Citation: Appl. Phys. Lett. 97, 142503 (2010); doi: 10.1063/1.3498009

View online: https://doi.org/10.1063/1.3498009

View Table of Contents: http://aip.scitation.org/toc/apl/97/14

Published by the American Institute of Physics

\section{Articles you may be interested in}

Phase locking of vortex based spin transfer oscillators to a microwave current

Applied Physics Letters 98, 132506 (2011); 10.1063/1.3565159

Modulation bandwidth of spin torque oscillators under current modulation

Applied Physics Letters 105, 152401 (2014); 10.1063/1.4898093

Perfect and robust phase-locking of a spin transfer vortex nano-oscillator to an external microwave source Applied Physics Letters 104, 022408 (2014); 10.1063/1.4862326

Nanoconstriction-based spin-Hall nano-oscillator

Applied Physics Letters 105, 172410 (2014); 10.1063/1.4901027

Injection locking of tunnel junction oscillators to a microwave current

Applied Physics Letters 98, 182503 (2011); 10.1063/1.3587575

The design and verification of MuMax3

AIP Advances 4, 107133 (2014); 10.1063/1.4899186

\section{HDDEN}

\section{Instruments for Advanced Science}

Contact Hiden Analytical for further details: W www.HidenAnalytical.com E info@hiden.co.uk CLICK TO VIEW our product catalogue

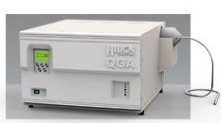

Gas Analysis

dynamic measurement of reaction gas streams cataysis and thermal analysis - molecuar beam sudes fermentation, envirormental and ecological studies

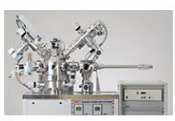

Surface Science

UHVTPD

SIMS

end point detection in in beam etch elemental imaging -surface mapping

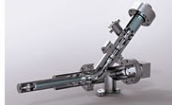

Plasma Diagnostics

plasma source characterization

etch and depositon process reacton

analysis of neutral and radical species

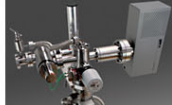

Vacuum Analysis

partial pressure measurement and control 


\title{
Injection locking of the gyrotropic vortex motion in a nanopillar
}

\author{
R. Lehndorff, ${ }^{1}$ D. E. Bürgler, ${ }^{1, a)}$ C. M. Schneider, ${ }^{1}$ and Z. Celinski ${ }^{2}$ \\ ${ }^{1}$ Institute of Solid State Research, Electronic Properties (IFF-9) and JARA-FIT, Research Center Jülich \\ GmbH, D-52425 Jülich, Germany \\ ${ }^{2}$ Center for Magnetism and Magnetic Nanostructures, University of Colorado at Colorado Springs, \\ Colorado 80918, USA
}

(Received 29 June 2010; accepted 17 September 2010; published online 5 October 2010)

\begin{abstract}
Spin-torque oscillators (STOs) are a promising application for the spin-transfer torque effect. The major challenge lies in pushing the STO's microwave output power to useful levels, e.g., by operating an array of STOs in a synchronized, phase-locked mode. Our experiment on metallic, giant magnetoresistance-type nanopillars focuses on the influence of external high-frequency signals on the current-driven vortex dynamics and demonstrates the injection locking of the gyrotropic mode. We find a gap of about three orders of magnitude between the high-frequency power emitted by one oscillator and the power needed for phase-locking. () 2010 American Institute of Physics.
\end{abstract} [doi:10.1063/1.3498009]

The action of spin-polarized currents onto a magnetic system - the spin-transfer torque-offers a novel handle on magnetization dynamics. One of the potential applications is seen in the so-called spin-torque oscillators (STOs). These are pillar-shaped structures with a "fixed" magnetic layer acting as a polarizer for the electric current and a second "free" magnetic layer the magnetization of which oscillates under the impact of the spin-polarized current. The magnetoresistive response due to giant or tunneling magnetoresistance (GMR, TMR) in the magnetic layered structure turns the nanometer-scaled STO structures into sources for highfrequency (HF) signals in the gigahertz range. The frequency is tunable via the direct current (dc) and the external magnetic field. However, the HF output power of an STO is presently far too low for applications. This is particularly true for metallic, GMR-based STOs due to their low MR ratio of only a few percent yielding output powers in the picowatt to low nanowatt regime. TMR-based STOs with TMR ratios of the order of $100 \%$ have been shown to generate about $1 \mu \mathrm{W}$ of output power. ${ }^{1}$

Ongoing research aims at strategies to significantly increase the output power. To advance on this, each STO should emit the maximum achievable power. We have recently pointed out a way to maximize the power by operating an STO in the vortex mode, i.e., by exciting with dc current the gyrotropic vortex mode instead of the precessional modes of the quasiuniform, single-domain state. ${ }^{2}$ A further advantage of vortex STOs is that they intrinsically operate in zero external field. This can also be achieved in singledomain STOs, however, only when using carefully selected layer structures or geometries, ${ }^{3-5}$ which impose design restrictions.

Generally, the excitation of an array of STOs in a synchronized, phase-locked manner is believed to deliver a significant power increase, as $N$ coherently coupled STOs emit up to the $N^{2}$-fold power. The STO-STO coupling can be achieved in two ways. First, by spin waves in a common ferromagnetic layer, as has been already demonstrated for two ${ }^{6-8}$ and four ${ }^{9}$ STOs. Due to the fast decay of spin waves this interaction is short-range and requires a STO-STO sepa-

${ }^{a)}$ Electronic mail: d.buergler@fz-juelich.de. ration of the order of the STO's diameter, i.e., clearly below $1 \mu \mathrm{m}$. The second coupling mechanism via microwaves propagating in common electrodes ${ }^{10,11}$ permits a larger STO separation because electrical microwave signals propagate with negligible losses over long distances. Experimentally, this situation has been addressed by injection locking experiments, which study the interaction of a STO with an externally applied HF signal in order to investigate conditions for synchronization and phase-locking. Rippard et al. ${ }^{12}$ have shown the injection locking of a single-domain STO.

In this letter, we demonstrate phase-locking of the dc current-driven gyrotropic vortex motion in a vortex STO to external HF signals, and derive from the HF amplitude dependence of the locking criteria for the required STO output power for synchronization.

We fabricate our samples from epitaxial (001)-oriented multilayers of Ag $150 \mathrm{~nm} / \mathrm{Fe} 2 \mathrm{~nm} / \mathrm{Ag} 6 \mathrm{~nm} / \mathrm{Fe} 20 \mathrm{~nm} / \mathrm{Au} 50$ $\mathrm{nm}$ grown by molecular beam epitaxy. ${ }^{13}$ Cylindrical pillars of $230 \mathrm{~nm}$ diameter are fabricated by means of optical and electron beam lithography combined with ion beam etching, reactive ion etching, and lift-off. ${ }^{13}$ The upper $20 \mathrm{~nm}$ thick Fe layer and the Au $50 \mathrm{~nm}$ cap layer form the pillar while the remaining layers $\mathrm{Ag} 150 \mathrm{~nm} / \mathrm{Fe} 2 \mathrm{~nm} / \mathrm{Ag} 6 \mathrm{~nm}$ constitute the $15 \mu \mathrm{m}$ wide bottom electrode [inset of Fig. 1(a)]. The electrode layout allows for contacting by two microwave probes via coplanar waveguides. One is used to inject a dc current and an HF current $\left(f_{\text {ext }}\right)$ from a network analyzer. The two currents are combined in a bias-T. The second probe is connected to a $30 \mathrm{~dB}$ amplifier and a spectrum analyzer (see top of Fig. 2). The experiments were conducted at $300 \mathrm{~K}$ in a microwave probe station with an in-plane magnetic field of up to $300 \mathrm{mT}$.

In Fig. 1(a) we compare measurements of the MR in current-perpendicular-plane (CPP) geometry in an in-plane magnetic field (CPP-GMR) with corresponding micromagnetic simulations. The nanodisk has two static magnetic states. The quasiuniform onion state occurs when the field is reduced after saturation and the vortex state nucleates at low fields after field reversal. The magnetization of the extended layer is saturated along an easy axis for fields larger than 20 $\mathrm{mT}$. From this we can identify the magnetization states of the $\mathrm{Fe}$ layers at various fields. ${ }^{2}$ On this basis we know how to 

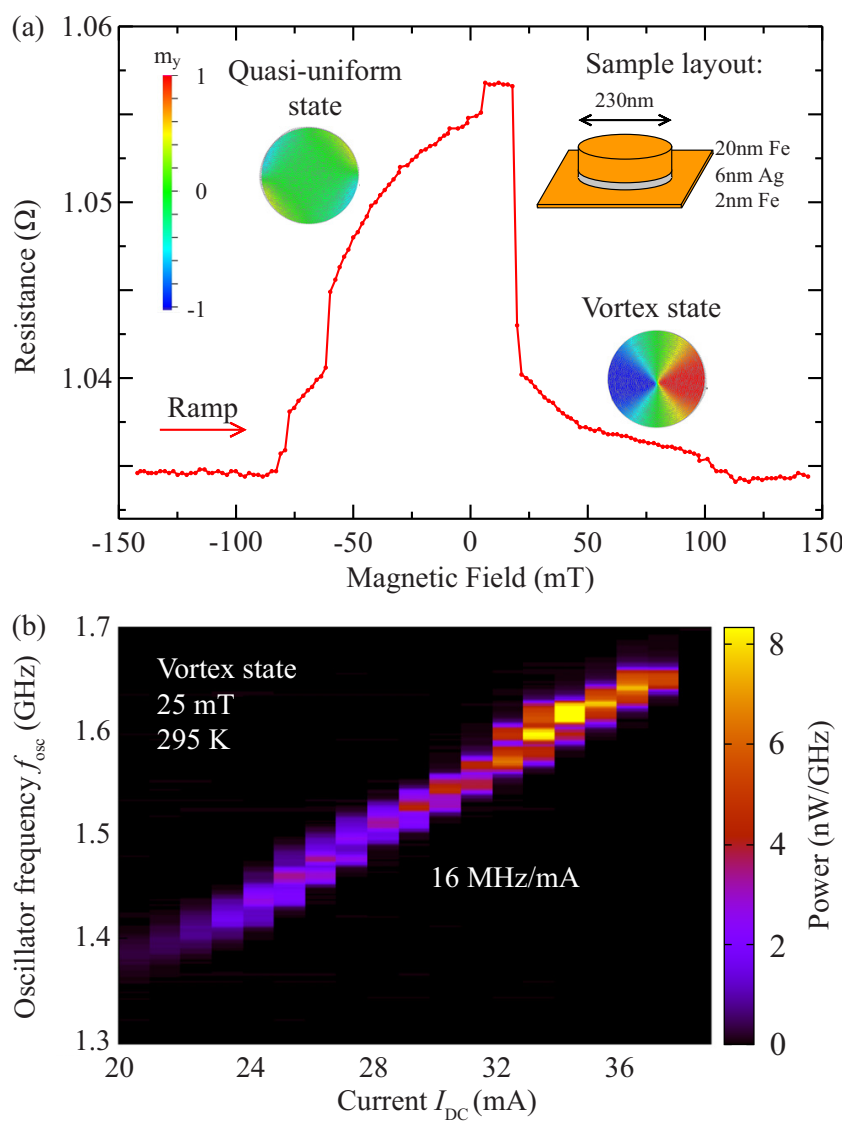

FIG. 1. (Color online) (a) CPP-GMR in comparison with micromagnetic calculations of the magnetization structure in the nanodisk (inset: sample layout). (b) Power spectra of the current-induced gyrotropic mode as a function of dc current $I_{\mathrm{dc}}$

prepare a vortex state in the nanodisk while, at the same time, the magnetization of the extended layer is saturated along a magnetic easy axis of bcc Fe, e.g., by applying +25 mT after negative saturation.

Applying positive dc currents (i.e., electron flow from the nanomagnet to the extended layer) in the range of 20 to $39 \mathrm{~mA}$ the vortex state is excited by spin-transfer torque into the gyrotropic mode ${ }^{14}$ that shows up as an HF voltage signal derived from the GMR [Fig. 1(b)]. Its frequency $f_{\text {osc }}$ increases linearly with the current at a rate of $16 \mathrm{MHz} / \mathrm{mA}^{2}$ These findings are in agreement with a mechanism for spintransfer induced gyrotropic motion of a vortex in a spinvalve nanostructure with an in-plane magnetized polarizer proposed by Khvalkovskiy et al. ${ }^{15}$ The model assumes a nonuniform, i.e., symmetry-breaking magnetization of the polarizer. An alternative mechanism may be related to the strong angular asymmetry of the spin-transfer torque in Fe/ $\mathrm{Ag} / \mathrm{Fe}(001)$ structures, ${ }^{16}$ which also breaks the symmetry in the sample plane. ${ }^{17}$

Adding a HF component to the driving current we are able to demonstrate phase-locking of the dc current-induced gyrotropic motion to electric HF signals even if they are slightly out of tune. Figure 2(a) shows a measurement, where the vortex was excited by a current of $32 \mathrm{~mA}$ to a frequency of $f_{\text {osc }}=1.518 \mathrm{GHz}$ while an external signal of $-17 \mathrm{~dB}$ m or $20 \mu \mathrm{W}$ was swept from $f_{\text {ext }}=1.35$ to $1.7 \mathrm{GHz}$. This leads to a clear shift in the vortex frequency between 1.42 and 1.61 $\mathrm{GHz}$. From 1.46 to $1.57 \mathrm{GHz}$ the vortex frequency $f_{\text {osc }}$ is completely locked to the external signal $f_{\text {ext }}$ and is thus masked by it. Upon an amplitude increase [Fig. 2(b)] the

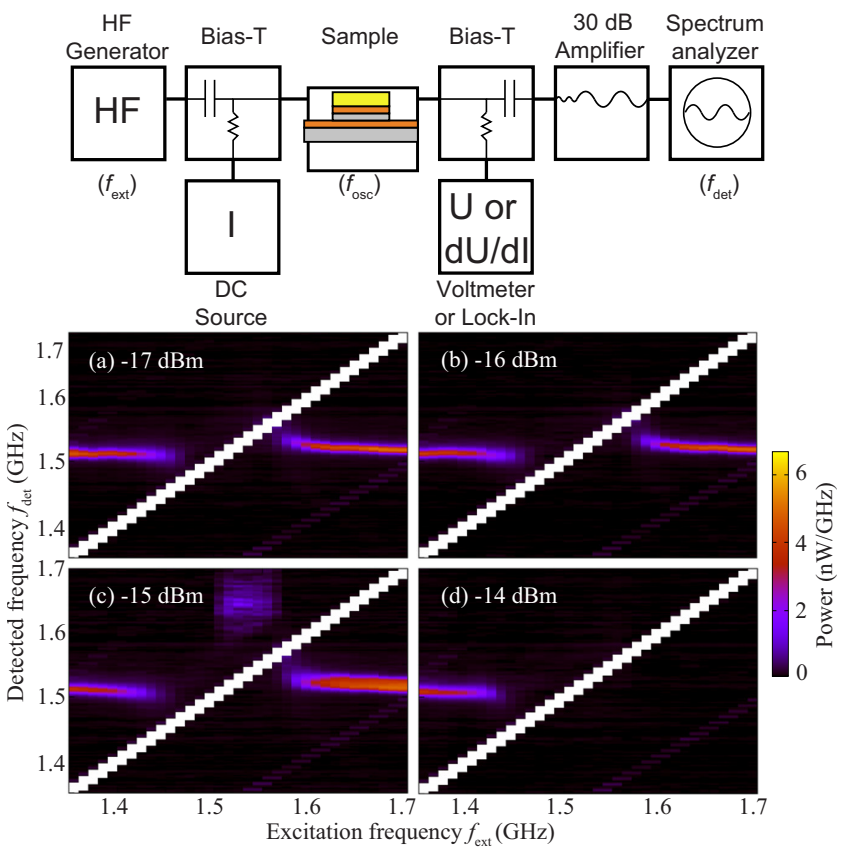

FIG. 2. (Color online) Measurement setup for injection locking and power spectra $\left(f_{\text {det }}\right.$, vertical axis) of the current-induced gyrotropic mode as a function of HF excitation frequency ( $f_{\text {ext }}$, horizontal axis) measured for different excitation amplitudes as indicated. The intensity at $f_{\text {ext }}$ exceeds the color scale and appears as diagonal white lines.

phase-locking ranges widen without qualitative changes. At $-15 \mathrm{~dB} m$ [Fig. 2(c)] an additional signal of $1.65 \mathrm{GHz}$ shows up in the right-hand part of the phase-locked regime and the signal shape and intensity changes for external frequencies $f_{\text {ext }}$ above $1.6 \mathrm{GHz}$. The origin of these features is not clear. Micromagnetic simulations are required to relate them to modifications of the gyrotropic mode, e.g., periodic vortex core reversals, ${ }^{15}$ or the presence of additional excited modes at increased HF amplitude. Finally, for strongest excitations at $-14 \mathrm{~dB} \mathrm{~m}$ [Fig. 2(d)] the vortex signal is lost above $1.5 \mathrm{GHz}$ when sweeping through the locking regime from low to high $f_{\text {ext }}$. The dc resistance after completing the frequency sweep is larger than before, which-based on Fig. 1 -must be identified with the quasiuniform magnetization state. Thus, the vortex has been expelled from the sample while being phase-locked to the HF signal. This may happen, when the radius of the gyrotropic trajectory strongly increases due to the excitation and the vortex finally hits the boundary of the nanodisk.

In order to evaluate the synchronization behavior indepth we fit the frequency variation $f_{\text {osc }}$ in the forced vortex motion with the frequency $f_{\text {ext }}$ of the injected HF signal to a formula derived by Slavin and Tiberkevich for nonlinear oscillators as follows [Eq. (48b) of Ref. 18]:

$$
f_{\text {osc }}=f_{\text {ext }}+\operatorname{sign}\left(f_{0}-f_{\text {ext }}\right) \sqrt{\left(f_{0}-f_{\text {ext }}\right)^{2}-\Delta^{2}},
$$

where $f_{0}=f_{\mathrm{osc}}\left(I_{\mathrm{HF}}=0\right)$ is the frequency of the free-running vortex STO and $\Delta$ is the phase-locking range. An example of a fit is shown in the inset of Fig. 3. The main figure shows an increase in $\Delta$ with increasing amplitude of the external signal in accordance with the linear dependence predicted by Slavin and Tiberkevich. ${ }^{18}$ The conversion from HF power to $\mathrm{HF}$ current amplitudes $I_{\mathrm{HF}}$ is given by the impedance of the sample $(11 \Omega$ at $1.5 \mathrm{GHz})$ that we have measured with a network analyzer. However, the linear extrapolation to zero excitation amplitude (dashed line in Fig. 3) yields a nonva- 


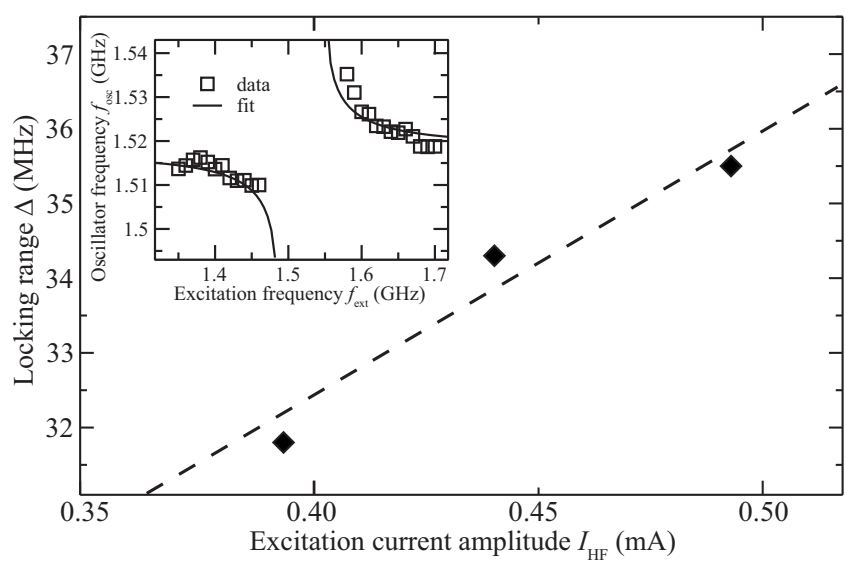

FIG. 3. Locking range $\Delta$ as a function of the external HF excitation amplitude. The dashed line is a linear fit. Inset: Fit (solid line) of Eq. (1) to the experimental locking behavior for $I_{\mathrm{dc}}=32 \mathrm{~mA}$ and $-16 \mathrm{~dB}$ m.

nishing locking range of about $18 \mathrm{MHz}$. This inconsistency may be due to the fact that the theoretical model assumes only a weak excitation. Our data indicates that the widening of the locking range $\Delta$ with the excitation amplitude proceeds slower for strong excitation.

Figure 4 shows the locking behavior of the vortex STO when excited by a fixed dc current of $32 \mathrm{~mA}$ and an external $1.55 \mathrm{GHz}$ signal of variable amplitude. For weak excitation the STO is not influenced by the HF signal and emits at its free-running frequency $f_{0} \approx 1.52 \mathrm{GHz}$. With increasing $\mathrm{HF}$ amplitude the STO tends to adjust to the external frequency and phase-locks for excitation amplitudes exceeding -18 $\mathrm{dB} \mathrm{m}$. The locking process is accompanied by a weakening and broadening of the STO signal. The HF signal generated by the magnetization dynamics in the pillar and the externally applied HF signal passing through the pillar are reaching the spectrum analyzer via a common pathway (righthand branch of the setup in Fig. 2). Therefore, we can directly determine the ratio between the power required for phase-locking and the power generated by the pillar from the measured spectra and obtain a ratio of about 3300 . The HF signal is attenuated in cables, connectors, the waveguides on the sample, and due to impedance mismatch, e.g., between leads and pillar. We characterize the sum of all these effects by measuring the transmission from the HF generator to the spectrum analyzer. Based on this transmission value and the symmetry of the setup we estimate the external HF power at $-18 \mathrm{~dB} m$ reaching the pillar to be of the order of $1.3 \mu \mathrm{W}$. Thus, the vortex STO generates roughly $0.4 \mathrm{nW}$ of microwave power. This low value is related to the low absolute

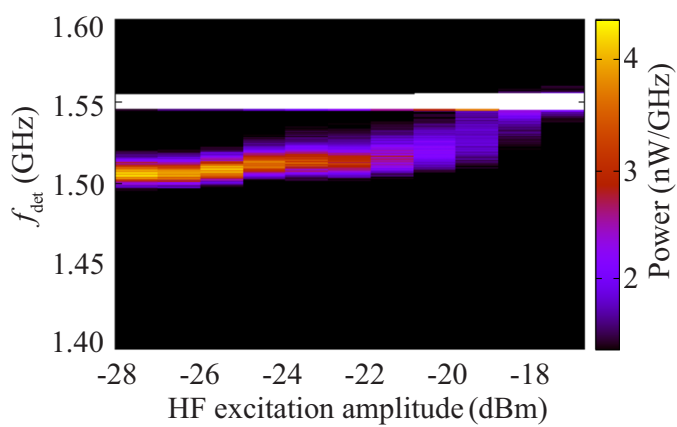

FIG. 4. (Color online) Locking behavior of a vortex STO as a function of the HF excitation amplitude at $f_{\mathrm{ext}}=1.55 \mathrm{GHz}$ and $I_{\mathrm{dc}}=32 \mathrm{~mA}$. Locking occurs at about $-18 \mathrm{~dB}$. resistance and low GMR ratio, which result in small resistance changes due to the magnetization dynamics. We emphasize that the physics of the vortex dynamics described in this letter is not affected by the weak conversion to output power.

Consequently, the output power of our GMR-based vortex STO is about three orders of magnitude too small to phase-lock another vortex STO. Under these conditions synchronization of an array of vortex STOs seems unlikely, unless the output power of each single STO is significantly increased, e.g., by employing highly spin-polarized ferromagnetic electrode materials (Heusler alloys ${ }^{19}$ ) or TMRbased structures with much higher MR ratios.

In conclusion, we demonstrated the possibility to phaselock the current-driven vortex motion in an STO to an external HF signal, being a prerequisite for the synchronization of vortex STOs. The relative locking range $2 \Delta / f_{0} \approx 5 \%$ is rather large and allows for a distribution of free-running frequencies in a STO array, which seems to be compatible with present fabrication technology. The power requirements for synchronization, however, call for STOs with much higher output power and for optimization of the synchronization efficiency, e.g., by appropriate phase control. ${ }^{20}$

We thank P. Grünberg for valuable discussions and R. Schreiber and F.-J. Köhne for technical support. This work was partly funded by the German academic exchange service (DAAD) and at UCCS by the NSF (Grant No. DMR0605629) and the ARO (Grant No. W911NF-04-1-0247).

${ }^{1}$ A. M. Deac, A. Fukushima, H. Kubota, H. Maehara, Y. Suzuki, S. Yuasa, Y. Nagamine, K. Tsunekawa, D. D. Djayaprawira, and N. Watanabe, Nat. Phys. 4, 803 (2008).

${ }^{2}$ R. Lehndorff, D. E. Bürgler, S. Gliga, R. Hertel, P. Grünberg, and C. M. Schneider, Phys. Rev. B 80, 054412 (2009).

${ }^{3}$ O. Boulle, V. Cros, J. Grollier, L. G. Pereira, C. Deranlot, F. Petroff, G. Faini, J. Barnas, and A. Fert, Nat. Phys. 3, 492 (2007).

${ }^{4}$ T. Devolder, A. Meftah, K. Ito, J. A. Katine, P. Crozat, and C. Chappert, J. Appl. Phys. 101, 063916 (2007).

${ }^{5}$ R. Lehndorff, D. E. Bürgler, A. Kakay, R. Hertel, and C. M. Schneider, IEEE Trans. Magn. 44, 1951 (2008).

${ }^{6}$ S. Kaka, M. Pufall, W. H. Rippard, T. J. Silva, S. E. Russek, and J. Katine, Nature (London) 437, 389 (2005).

${ }^{7}$ F. B. Mancoff, N. D. Rizzo, B. N. Engel, and S. Tehrani, Nature (London) 437, 393 (2005).

${ }^{8}$ M. R. Pufall, W. H. Rippard, S. E. Russek, S. Kaka, and J. A. Katine, Phys. Rev. Lett. 97, 087206 (2006).

${ }^{9}$ A. Ruotolo, V. Cros, B. Georges, A. Dussaux, J. Grollier, C. Deranlot, R. Guillemet, K. Bouzehouane, S. Fusil, and A. Fert, Nat. Nanotechnol. 4 528 (2009).

${ }^{10}$ J. Grollier, V. Cros, and A. Fert, Phys. Rev. B 73, 060409 (2006).

${ }^{11}$ B. Georges, J. Grollier, M. Darques, V. Cros, C. Deranlot, B. Marcilhac, G. Faini, and A. Fert, Phys. Rev. Lett. 101, 017201 (2008).

${ }^{12}$ W. H. Rippard, M. R. Pufall, S. Kaka, T. J. Silva, S. E. Russek, and J. A. Katine, Phys. Rev. Lett. 95, 067203 (2005).

${ }^{13}$ H. Dassow, R. Lehndorff, D. E. Bürgler, M. Buchmeier, P. A. Grünberg, C. M. Schneider, and A. van der Hart, Appl. Phys. Lett. 89, 222511 (2006).

${ }^{14}$ K. Y. Guslienko, B. A. Ivanov, V. Novosad, Y. Otani, H. Shima, and K. Fukamichi, J. Appl. Phys. 91, 8037 (2002).

${ }^{15}$ A. V. Khvalkovskiy, J. Grollier, N. Locatelli, Y. V. Gorbunov, K. A. Zvezdin, and V. Cros, Appl. Phys. Lett. 96, 212507 (2010).

${ }^{16}$ R. Lehndorff, D. E. Bürgler, A. Kakay, R. Hertel, and C. M. Schneider, Phys. Rev. B 76, 214420 (2007).

${ }^{17}$ V. Sluka and D. E. Bürgler (unpublished).

${ }^{18}$ A. Slavin and V. Tiberkevich, IEEE Trans. Magn. 45, 1875 (2009).

${ }^{19}$ T. Iwase, Y. Sakuraba, S. Bosu, K. Saito, S. Mitani, and K. Takanashi, Appl. Phys. Express 2, 063003 (2009)

${ }^{20}$ Y. Zhou, J. Persson, S. Bonetti, and J. Åkerman, Appl. Phys. Lett. 92, 092505 (2008). 